\title{
Extract of Dendropanax morbiferus H.Lév. Leaves Induces Apoptosis in Human Lung Carcinoma A549 Cells
}

\author{
Eun-Seon Yoo ${ }^{1}$, Sung-Hyun Kim ${ }^{1}$, Joong-Seok Woo ${ }^{1}$,Jae-Han Lee ${ }^{1}$, So-Hee Han ${ }^{1}$, Soo-Hyun Jung ${ }^{1}$, Sae Man Kim², Eun Gee Kim², Kwang \\ Joong Kim ${ }^{3}$ and Ji-Youn Jung ${ }^{*}$ \\ ${ }^{1}$ Department of Companion and Laboratory Animal Science, Kongju National University, Daehak-ro, Yesan-eup, Yesan-gun, Chungcheongnam 32439, Republic \\ of Korea \\ ${ }^{2}$ DPBioPharm, Wolmuncheon-ro, Wabu-eup, Namyangju-si, Gyeonggi-do 12213, Republic of Korea \\ ${ }^{3}$ ELDO Healthcare Co. Ltd., Gwanpyeong-ro, Dongan-gu, Anyang-si, Gyeonggi-do 14066, Republic of Korea
}

\begin{abstract}
Dendropanax morbiferus H.Lév. has been used in traditional medicine for the treatment of skin diseases, migraine, dysmenorrhea and other maladies. Recent studies have reported that extract of Dendropanax morbiferus H.Lév. has an anti-tumor effect on several types of cancer cells in vitro. In this study, we investigated the anticancer effects and molecular mechanisms of extracts of Dendropanax morbiferus H. Lév. (DPL) in human lung carcinoma. In vitro studies were performed using the MTT assay, DAPI staining, flow cytometry and western blot. In vivo studies were performed on four-week-old female BALB/c nude mice using xenografts and oral administration, terminal deoxynucleotidyl transferase dUTP nick end labeling, immunohistochemistry, hematoxylin and eosin. The viability of A549 lung carcinoma cells assessed by MTT assay decreased in a concentration-dependent manner from $300 \mu \mathrm{g} / \mathrm{mL}$. DAPI staining revealed that DNA fragmentation significantly increased in a concentration-dependent manner, indicating apoptosis. Flow cytometry revealed that apoptosis was increased in a dose-dependent manner following treatment with DPL. Western blotting revealed that the protein levels of Bax, cleaved-poly (ADP-ribose) polymerase, and phospho-p38 increased while those of B-cell lymphoma 2 decreased. In addition, levels of phosphorylated ERK1/2 and p-c-Jun N-terminal kinases were significantly different from the control. We also investigated the in vivo effects of DPL on tumor growth. Tumor size decreased in cells treated with $500 \mathrm{mg} / \mathrm{kg}$ DPL compared with the control group in vivo. Apoptosis, assessed by terminal deoxynucleotidyl transferase dUTP nick end labeling, was significantly increased, and the inhibitory effect on tumors was confirmed. Immunohistochemistry staining showed increased expression of phospho-p38 in the $500 \mathrm{mg} / \mathrm{kg}$-treated group. The results indicate that DPL induced apoptosis through the p38 mitogen-activated protein kinase signaling pathway in A549 cells.
\end{abstract}

\section{Introduction}

The incidence of cancer and the number of deaths from cancer are continually increasing, and cancer is the leading cause of death in South Korea [1]. Lung cancer is the most prevalent cancer following stomach cancer, colorectal cancer, and thyroid cancer, comprising $22.8 \%$ of total cancer deaths [2]. Lung cancer can be classified into small cell lung carcinoma (SCLC) and non-small cell lung carcinoma (NSCLC); the latter represents $85-90 \%$ of the total cases of lung cancer [3]. NSCLC is resistant to radiotherapy and chemotherapy, the most commonly used treatments, and occurs at a later stage, which makes surgical treatment difficult. For these reasons, the 5-year survival rate of NSCLC is less than $15 \%$ [4]. There are many therapies for the treatment of lung cancer, including surgery, radiotherapy, chemotherapy, and targeted therapy, but there have been no studies indicating the optimal therapy, suggesting the need for new strategies [5]. The study of natural materials is increasing as a source of future cancer treatments because compounds extracted from natural sources have contributed significantly to the development of new drugs; more than $60 \%$ of approved drugs are derived from natural compounds, as shown by the investigation of chemotherapeutic agents and their sources [6].

Dendropanax morbiferus H.Lév. is an evergreen broad-leaved tree in the Araliaceae family found in the Jeollanam-do coastal area and Jeju island. Its yellow sap is used to paint and coat the surface of wooden crafts. D. morbiferus extracts have been used to improve blood circulation, boost immunity, and treat diabetes [7]. Recent reports on
D. morbiferus confirmed the presence of 32 substances in the tree using capture compound mass spectrometry, of which $\beta$-selinene is the most abundant, followed by capnellane-8-one, a type of sesquiterpene with two annuli [8]. The extract of D. morbiferus has been reported to exhibit anti-tumor [9], antioxidant, whitening [10,11], and anti-inflammatory activities [12], and it is used to treat diabetes [13]. However, there is no research on whether it exerts anti-tumor effects in vivo. Especially, it has not found to have antitumor effects in vivo in human lung carcinoma in vivo.

Apoptosis plays a role in homeostasis by maintaining cells during development and aging, and in tissues; it is initiated by a defensive mechanism following damage to the immune system caused by a disease or harmful substances. A variety of harmful irritations, such as heat, radiation, or low oxygen, can induce apoptosis at a low level and necrosis at a high level. Apoptosis occurs either exogenously or endogenously. When the caspase cascade is initiated, the DNA is

${ }^{*}$ Correspondence to: Ji-Youn Jung, Department of Companion and Laboratory Animal Science, Kongju National University, Yesan-eup, Yesan-gun, Chungcheongnam-do, 32439, Koera, Tel: +82-41-330-1526; Fax: +82-41-3301529; E-mail: wangza@kongju.ac.kr

Key words: apoptosis, A549 cells, Dendropanax morbiferus H.Lév., human lung carcinoma, 38 MAPK pathway

Received: May 14, 2021; Accepted: May 24, 2021; Published: May 31, 2021 
fragmented, the cytoskeleton and nucleoproteins are decomposed, and proteins are cross-linked to form an apoptotic body, which is finally engulfed by a phagocyte [14]. Among apoptosis-inducing mechanisms, the mitogen-activated protein kinase (MAPK) pathway plays an important role in tumor formation; activated MAPK regulates cell growth, differentiation, apoptosis, and proliferation. Recently, small molecule inhibitors targeting this pathway have been developed and clinical studies are ongoing [15].

This study demonstrated induction of apoptosis by the extract of D. morbiferus leaves (DPL) through the MAPK pathway in A549 cells, a NSCLC cell line, and examined the effect of the extract on tumor growth in vivo.

\section{Material and methods}

\section{Chemicals, Drugs, and Antibodies}

The A549 lung carcinoma cells (No. 10185) used in this study were purchased from the Korean Cell Line Bank (Seoul, Korea). The WBF344 normal hepatic cells (No. JCRB0193) used in this study were purchased from the Japanese Collection of Research Bioresources Cell Bank (Osaka, Japan). RPMI-1640 was purchased from Welgene (Gyeongsan, Korea), and streptomycin/penicillin and fetal bovine serum (FBS) were purchased from Gibco BRL (Grand Island, NY, USA). Experimental reagents were purchased from Sigma-Aldrich Co. (St. Louis, MO, USA). Anti-rabbit IgG, anti-Bcl-2, anti-Bax, antipoly (ADP-ribose) polymerase (PARP), anti-phospho (p)-extracellular signal-regulated kinase (ERK) $1 / 2$, anti-p-c-Jun N-terminal kinase (JNK), anti-p-p38, anti-ERK1/2, anti-JNK, anti-p38, and anti- $\beta$-actin were purchased from Cell Signaling Technology (Danvers, MA, USA).

\section{Cell culture}

A549 lung carcinoma and normal WB hepatic cells were cultured in RPMI-1640 medium containing 5\% FBS and 1\% penicillin/ streptomycin at $37^{\circ} \mathrm{C}$ in a humidified $5 \% \mathrm{CO}_{2}$ atmosphere. When the cells reached $80-90 \%$ confluence, they were sub-cultured, and the culture medium was replaced every 2-3 days.

\section{Plant materials and extraction}

DPL were purchased from Sinbo Pharmacy (Seoul, Korea). The origin of D. morbiferus was Jeju Island, Korea. DPL (100 g) were crushed, fermented with $70 \%$ ethanol and deionized water at a ratio of 4:6 for 10 days at room temperature, and then extracted with hot water. The extracted solution was filtered using Whatman No. 1 disk paper. The extracted liquid was vacuum-evaporated in a rotary evaporator (R220; BUCHI corporation, New Castle, DE, USA) to obtain the extract through reheating [16]. The extract was then mixed with ethanol and refrigerated.

\section{3-(4,5-dimethylthiazol-2-yl)-2,5-diphenyltetrazolium bromide (MTT) Assay}

The MTT assay was conducted to determine whether the viability of A549 lung carcinoma and WB hepatic cells decreased following exposure to DPL. A total of $2 \times 10^{4}$ cells/mL was cultured on a 96well plate; after $24 \mathrm{~h}$, the culture medium was discarded. WB cells were added to culture medium containing 0-1000 $\mu \mathrm{g} / \mathrm{mL} \mathrm{DPL}$, while A549 cells were added to culture medium containing 0-500 $\mu \mathrm{g} / \mathrm{mL}$ DPL and cultured for $24 \mathrm{~h}$. After $24 \mathrm{~h}$, the culture medium containing DPL was removed, MTT solution $(1 \mathrm{mg} / \mathrm{mL})$ was added, and cells were cultured in a $\mathrm{CO}_{2}$ incubator for $1 \mathrm{~h} 30 \mathrm{~min}$. The MTT solution was removed after $1 \mathrm{~h} 30 \mathrm{~min}$. To dissolve the formazan, $100 \mu \mathrm{L}$ DMSO was added, and absorbance was measured at $595 \mathrm{~nm}$ with an ELISA reader (Bio-Rad Laboratories, Hercules, CA, USA).

\section{4',6-diamidino-2-phenylindole (DAPI)}

DAPI staining was conducted to demonstrate whether the decrease in viability of A549 cells was caused by apoptosis. A549 cells were divided into 60 dishes with $2 \times 10^{5}$ cells $/ \mathrm{mL}$ per dish and cultured for $24 \mathrm{~h}$. After $24 \mathrm{~h}$, the culture medium was removed, and culture medium containing DPL $(0,200$, and $300 \mu \mathrm{g} / \mathrm{mL})$ was added followed by culture for another $24 \mathrm{~h}$. Cells were washed twice with phosphate-buffered saline (PBS) and fixed in 4\% paraformaldehyde solution for $15 \mathrm{~min}$. Then, the paraformaldehyde was removed, cells were washed with PBS, and DAPI solution $(2 \mathrm{~mL})$ was added. DAPI-positive cells were observed at 200× magnification through an optical microscope (Zeiss fluorescence microscope, Carl Zeiss, Thornwood, NY, USA).

\section{Flow cytometry}

To analyze apoptosis, A549 cells were cultured in medium containing DPL $(0,200$, and $300 \mu \mathrm{g} / \mathrm{mL})$ in a $25 \mathrm{~cm}^{2}$ flask for $24 \mathrm{~h}$. Following $24 \mathrm{~h}$ of incubation, trypsin-EDTA was used to suspend the cells, which were then centrifuged at $1200 \mathrm{rpm}$ for $5 \mathrm{~min}$ at $4^{\circ} \mathrm{C}$. The resulting pellet was resuspended at $1 \times 10^{6}$ cells $/ \mathrm{mL}$ with $1 \times$ annexin $\mathrm{V}$ binding buffer. Fluorescein isothiocyanate-conjugated annexin- $\mathrm{V}$ and phycoerythrin-conjugated propidium iodide were added according to the manufacturer's instructions of the annexin $\mathrm{V}$ staining kit (BD Pharmingen, San Diego, CA, USA). Cells were incubated for $15 \mathrm{~min}$ and analyzed using flow cytometry.

\section{Western blotting}

Western blotting was conducted to assess the expression of apoptosis-related proteins. A549 cells were cultured for $24 \mathrm{~h}$ in culture medium containing DPL $(0,200$, and $300 \mu \mathrm{g} / \mathrm{mL})$ in a $75 \mathrm{~cm}^{2}$ flask, and the cells were suspended using trypsin-EDTA and centrifuged at $1200 \mathrm{rpm}$ for $5 \mathrm{~min}$ at $4^{\circ} \mathrm{C}$. The resulting pellets were lysed in lysis buffer (Invitrogen, Carlsbad, CA, USA) and centrifuged at 13,000 rpm for $5 \mathrm{~min}$ at $4^{\circ} \mathrm{C}$ to obtain whole-cell lysates. The concentration of the extracted protein was determined using a Bradford protein assay kit (Bio-Rad Laboratories), and proteins were separated by $5-12 \%$ sodium dodecyl sulfate polyacrylamide gel electrophoresis and transferred to a nitrocellulose membrane. The membrane was blocked for $1 \mathrm{~h}$ with Tris-buffered saline (TBS) containing 5\% non-fat dry milk and $0.1 \%$ Tween-20. After blocking, the membranes were incubated with antiBax, anti-Bcl-2, anti-PARP, anti-ERK1/2, anti-JNK, anti-p38, anti-pERK1/2, anti-p-JNK, anti-p-p38, and anti- $\beta$-actin antibodies overnight at $4^{\circ} \mathrm{C}$ with gentle shaking. After washing with TBS containing $0.1 \%$ Tween-20, membranes were incubated with horseradish peroxidaseconjugated secondary antibodies for $1 \mathrm{~h}$ at room temperature. After washing, bands were visualized using enhanced chemiluminescence detection reagents (Pierce, Rockford, IL, USA) according to the manufacturer's instructions. The density of each band was measured quantitatively using the ImageJ Launcher imaging program (provided by NCBI).

\section{Xenografts}

Xenografts were performed to examine whether the in vitro antitumor effects also occurred in vivo. BALB/c nude mice are commonly used in anticancer studies involving tumor models. Four-week-old female BALB/c nude mice were purchased from Nara Biotec (Seoul, Korea). Animal experiments were performed in accordance with the Guidelines for the Care and Use of Animals of the Kongju National 
University Animals Care Committee (approval no. KNU_2019-02, Yesan, Korea). This animal experiment was conducted according to the same protocol used in previously reported experiments $[17,18]$. The mice were raised under a $12 \mathrm{~h}$ light cycle and controlled temperature $\left(23 \pm 3^{\circ} \mathrm{C}\right)$ and humidity $(40 \pm 10 \%)$. The mice had ad libitum access to pelleted feed and water and were housed in isolated, ventilated polycarbonate cages ( $\leq$ three mice per cage). A549 cells cultured at $37^{\circ} \mathrm{C}$ with $5 \% \mathrm{CO}_{2}$ were suspended using trypsin-EDTA and centrifuged (1200 rpm, $5 \mathrm{~min}, 4^{\circ} \mathrm{C}$ ). The pellet was washed twice and re-suspended in RPMI-1640 culture medium. From $0.2 \mathrm{~mL}$ of culture medium, $1 \times$ $10^{7}$ cells/mL were injected subcutaneously into both shoulders of the donor nude mice. Seven days after injection, A549 cells growing under the skin of nude mice established tumors. When the diameter was 3-4 $\mathrm{mm}$, ten mice weighing $20 \mathrm{~g}$ were randomly each assigned to both the control group [DW (distilled water)] and the $500 \mathrm{mg} / \mathrm{kg}$ DPL group $(n=5)$. Treatment was administered orally five times a week for 22 days. During the administration period, the general condition of the mice was examined, and the size of the tumors on both shoulders of the mice was measured by Vernier calipers (Mitutoyo Corporation; Kanagawa, Japan). The size and body weight of tumors were measured twice a week. The mice were sacrificed by $\mathrm{CO}_{2}$ inhalation at 22 days after treatment and the tumors were weighed in the laboratory. Tumor volume was calculated as follows: tumor volume $\left(\mathrm{mm}^{3}\right)=0.5 \times$ length $\times$ width $^{2}$. A portion of the tumor was embedded in paraffin and used for terminal deoxynucleotidyl transferase dUTP nick end labeling (TUNEL) and immunohistochemical analyses.

\section{TUNEL assay}

The TUNEL assay was conducted to demonstrate the induction of apoptosis in tumors. Apoptotic cell death was quantified using a Promega DeadEnd Colorimetric TUNEL system kit according to the manufacturer's instructions (Promega Corp., Madison, WI, USA). Briefly, tumor tissues were fixed in $10 \%$ formalin overnight and embedded in paraffin. The blocks were then cut into 5 - $\mu \mathrm{m}$-thick slices. The tissue sections attached to microscopic slides were deparaffinized by immersion in xylene and the slides were washed with 100\% ethanol. The samples were rehydrated by sequential immersion in a graded ethanol series $(95,85,70$, and $50 \%)$. The tumor sections were visualized using 3,3'-diaminobenzidine (DAB) solution, treated with mounting reagent, and observed under a microscope (BX41; Olympus Co., Tokyo, Japan) at $200 \times$ magnification. TUNEL-positive cells were counted to determine the number of cells in which apoptosis was induced.

\section{Immunohistochemistry}

Embedded paraffin sections were sequentially dipped in xylene and alcohol solution to deparaffinize and then rinsed with water. Sections were then incubated overnight at $4^{\circ} \mathrm{C}$ with p-p38 antibody and cultured for $1 \mathrm{~h}$ at room temperature with peroxidase-conjugated goat anti-rabbit antibody. Samples were then visualized with DAB solution, mounted, and observed using an optical microscope (200x).

\section{Histological Examination}

To evaluate organ toxicity induced by administration of DPL, livers and kidneys of mice were removed, fixed in 10\% formalin, and embedded in paraffin. The paraffin block was cut into 5 - $\mu \mathrm{m}$-thick sections, stained with hematoxylin and eosin, and observed using an optical microscope $(200 \times)$.

\section{Statistical Analysis}

All experiments were performed three times, and the results are presented as the mean \pm standard deviation. Differences between the means of the DPL and control group were evaluated by one-way analysis of variance and Dunnett's t-test. Differences with ${ }^{\star} P<0.05$ and ${ }^{*} P<0.01$ were considered statistically significant.

\section{Results}

\section{Administration of DPL decreased the viability of A549 cells}

The MTT assay was conducted to examine whether the viability of A549 lung carcinoma cells was reduced following administration of DPL. Before adding DPL to A549 cells, normal WB hepatic cells were treated with $0-1000 \mu \mathrm{g} / \mathrm{mL}$ DPL for $24 \mathrm{~h}$ and cell viability was measured. When $0-1000 \mu \mathrm{g} / \mathrm{mL}$ DPL was added to WB cells, the viability rate was similar to that of the control group treated with $0-500 \mu \mathrm{g} / \mathrm{mL}$ DPL; however, viability decreased following treatment of DPL at concentrations of 600 $\mu \mathrm{g} / \mathrm{mL}$ and higher. Therefore, $0,100,200,300,400$, and $500 \mu \mathrm{g} / \mathrm{mL}$ DPL were added to A549 cells; viability was measured following incubation for $24 \mathrm{~h}$. Viability decreased following treatment with $300-500 \mu \mathrm{g} / \mathrm{mL}$ DPL in a concentration-dependent manner; further experiments were conducted with 0,200 , and $300 \mu \mathrm{g} / \mathrm{mL}$ DPL (Figure 1). Thus, DPL was confirmed to reduce viability of A549 lung carcinoma cells.

\section{Administration of DPL induces apoptosis in A549 cells}

To demonstrate whether DPL induces apoptosis, DPL was added to A549 cells at concentrations of 0,200 , and $300 \mu \mathrm{g} / \mathrm{mL}$ for $24 \mathrm{~h}$; DAPI staining was used to measure morphological changes and flow cytometry was performed to measure apoptosis. DAPI staining revealed DNA fragmentation and apoptotic bodies; these effects increased in a concentration-dependent manner (Figure 2). Five sections were randomly chosen and 100 cells were selected to quantify the number of apoptotic cells. Following treatment with 0,200 , and $300 \mu \mathrm{g} / \mathrm{mL} \mathrm{DPL}$, the number the apoptotic cells increased by $2.6 \%, 15.0 \%$, and $25.0 \%$, respectively (Figure 2). When A549 cells were treated with 0, 200, and $300 \mu \mathrm{g} / \mathrm{mL}$ DPL, the number of apoptotic cells increased by $10.8 \%$, $15.9 \%$, and $22.7 \%$, respectively (Figure 3 ). These results indicate that DPL can induce apoptosis in A549 lung carcinoma cells.

\section{DPL regulates apoptosis-related proteins in A549 cells}

To examine the expression of apoptosis-related proteins, 0,200 , and $300 \mu \mathrm{g} / \mathrm{mL}$ DPL was added to A549 cells and western blotting was performed. Levels of the anti-apoptotic protein Bcl-2 decreased compared with the control group while levels of the pro-apoptotic proteins cleaved-PARP and Bax (Figure 4) increased. These results suggest that DPL reduced Bcl-2 and increased cleaved-PARP and Bax, inducing apoptosis.

\section{DPL regulates MAPK pathway proteins in A549 cells}

Western blotting was performed to examine whether apoptosis is induced through the MAPK pathway. When cells were treated with 0,200 , and $300 \mu \mathrm{g} / \mathrm{mL}$ DPL for $24 \mathrm{~h}$, the expression of $\mathrm{p}$-ERK1/2 and p-JNK was similar to that in the control group. On the other hand, levels of p-p38 increased in a concentration-dependent manner (Figure 4). These results imply that DPL induces apoptosis of A549 cells through the p38 MAPK pathway.

\section{DPL inhibits tumor growth in an animal model}

Xenografts were performed to examine whether the in vitro antitumor effects also occurred in vivo. A549 cells were transplanted in the subcutaneous tissue of $\mathrm{BALB} / \mathrm{c}$ nude mice, and 0 and $500 \mathrm{mg} / \mathrm{kg}$ DPL was orally administered five times a week for 3 weeks. Effects on 
WB

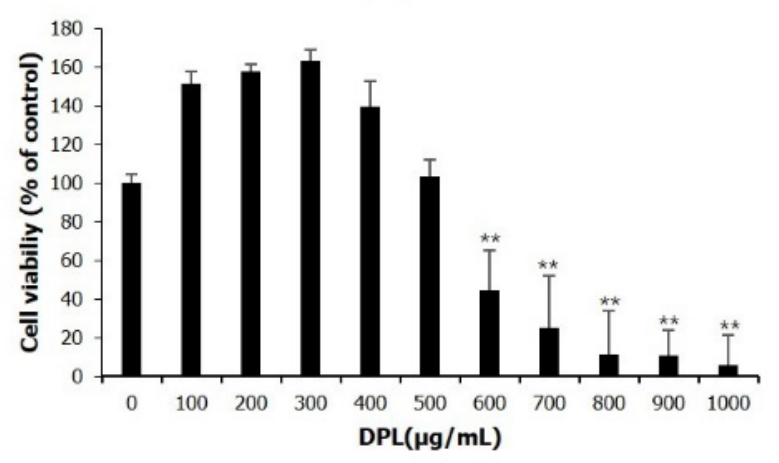

A549

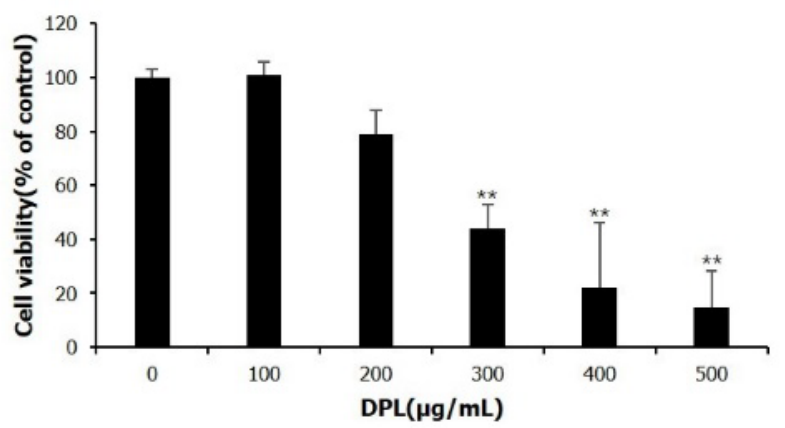

Figure 1. Effects of Dendropanax morbifera leaves (DPL) on the viability of WB and A549 cells Cell viability was measured using the MTT assay. The results are presented as the mean \pm standard deviation (SD) from three independent experiments performed in triplicate. Significance was determined by Dunnett's t-test. * $P<0.05, * * P<0.01$ versus control

A $\operatorname{DPL}(\mu \mathrm{g} / \mathrm{mL})$

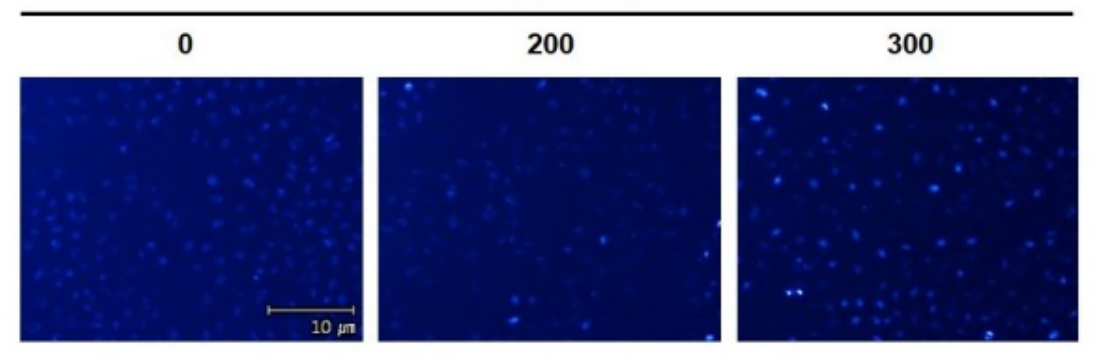

B

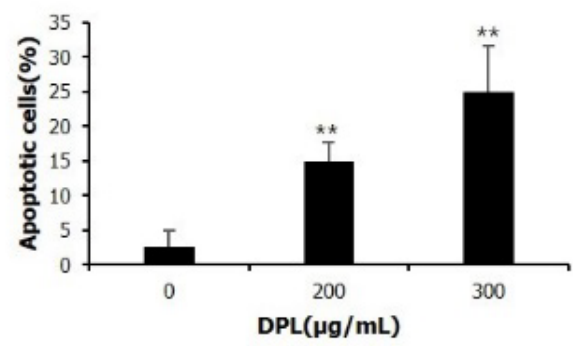

Figure 2. Effects of DPL on apoptotic bodies of A549 cells. (A) Chromatin condensation was examined using a fluorescence microscope (200×). (B) Quantification of apoptotic cells. Scale bar, $10 \mu \mathrm{m}$. Each bar represents the mean $\pm \mathrm{SD}$ calculated from three independent experiments. ${ }^{*} P<0.05, * * P<0.01$ versus control

A

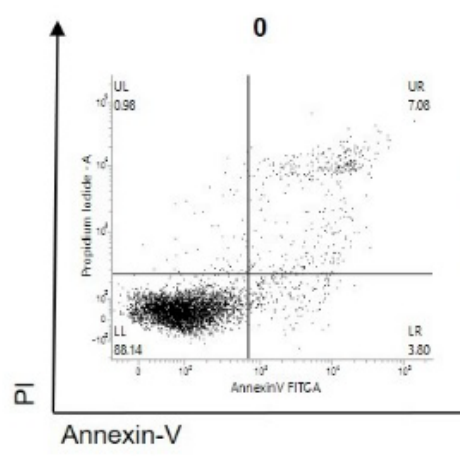

$\operatorname{DPL}(\mu \mathrm{g} / \mathrm{mL})$

200

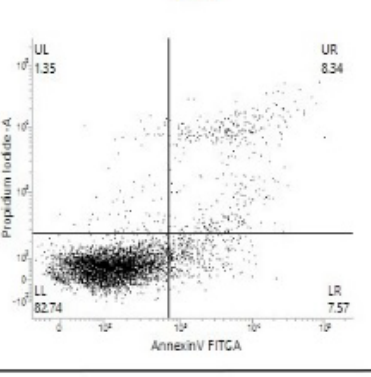

300

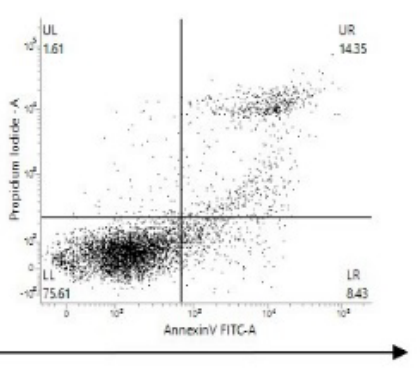

B

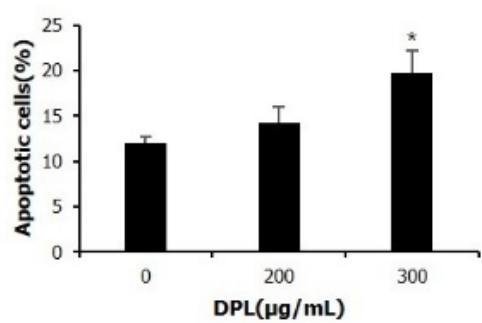

Figure 3. Effects of DPL on apoptosis of A549 cells. (A) Apoptotic cells were measured by flow cytometry. (B) Quantification of apoptotic cells. The results are presented as the mean \pm SD from three independent experiments performed in triplicate. ${ }^{*} P<0.05$ versus control

tumor growth were examined: tumor size was measured twice a week and tumor volume was compared to the control group, depending on DPL administration. Tumor growth was inhibited beginning at day 15 , and significantly inhibited, down to $40.6 \%$, by day 22 (Figure 5). Tumor weight was reduced $22.2 \%$ in the DPL-treated group relative to the control group (Figure 5). Body weight was similar between the DPLtreated and control groups (Figure 5). These results indicate that DPL can inhibit tumor growth in vivo.

\section{DPL induces apoptosis in A549 tumor tissue}

The anti-cancer effect of DPL on human lung carcinoma cells was examined using xenografts, and the induction of apoptosis in tumor tissue was observed using a TUNEL assay. Apoptosis was significantly increased in the group administered $500 \mathrm{mg} / \mathrm{kg}$ DPL compared with the control group (Figure 6). Thus, administration of DPL induces apoptosis of tumor tissue. 
A

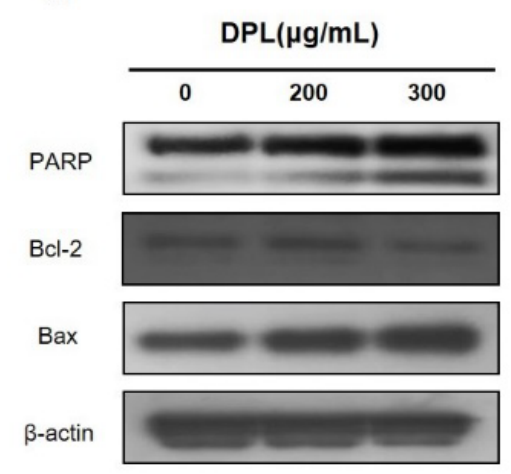

B

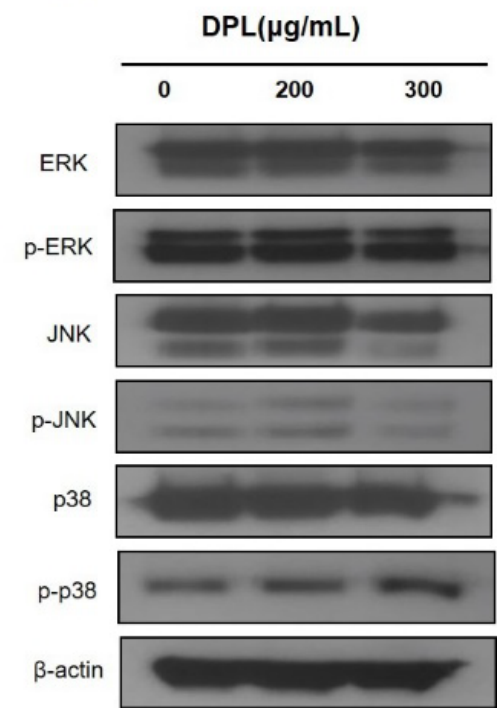

Figure 4. Effects of DPL on apoptotic protein levels of A549 cells. The cells were harvested to measure the levels of apoptotic proteins (A) and MAPK protein (B) by western blotting. $\beta$-actin was used as a loading control

A

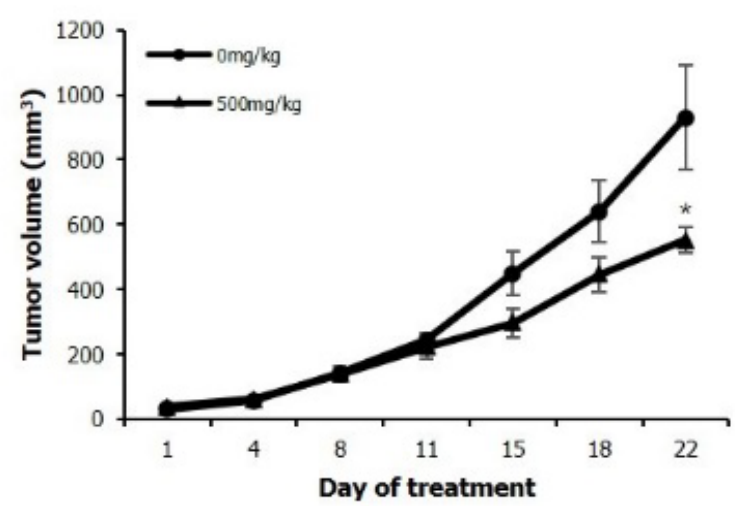

B

C
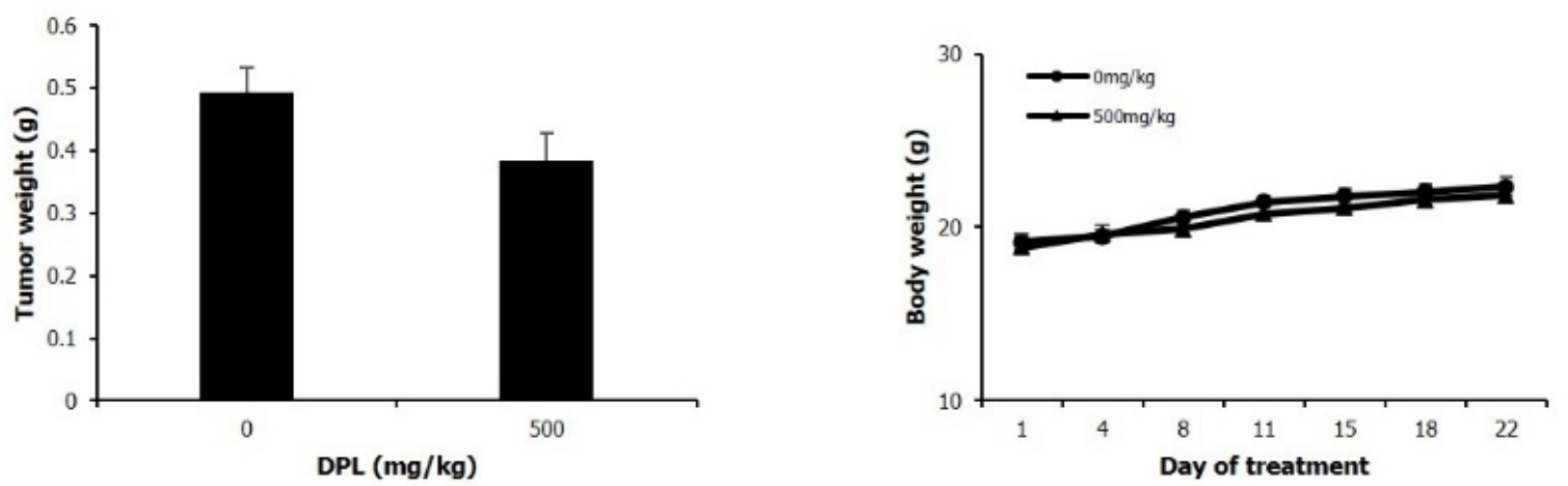

Figure 5. Effects of DPL on lung cancer tumor growth in tumor tissues. Nude mice bearing A549 cells as xenograft models were treated with DPL for 22 days, and tumor volume (A), weight (B) and body weight (C) were determined. Each value represents the mean \pm standard error. Significance was determined by Dunnett's t-test. $* P<0.05$, ** $P<0.01$ versus control 
A

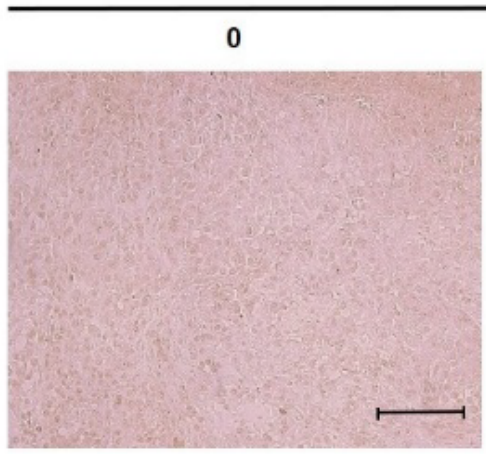

$\mathrm{DPL}(\mathrm{mg} / \mathrm{kg})$

500

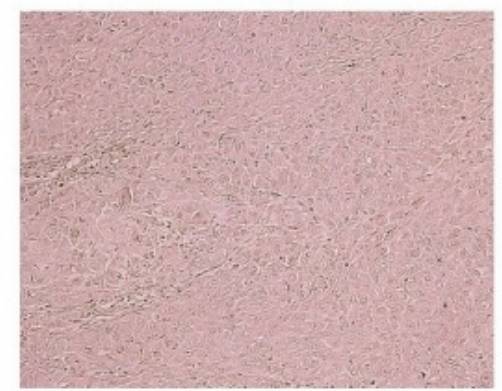

B

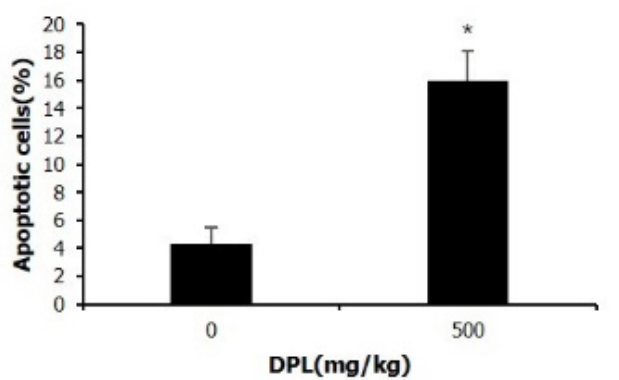

Figure 6. Effects of DPL on apoptosis in lung tumor tissues. Apoptosis was measured in tumor tissues using the TUNEL assay (A), and the number of apoptotic cells was quantified (B). Scale bar, $10 \mu \mathrm{m}$. Significance was determined by the Dunnett's t-test. $* P<0.05$ versus control

\section{$\mathrm{DPL}(\mathrm{mg} / \mathrm{kg})$}

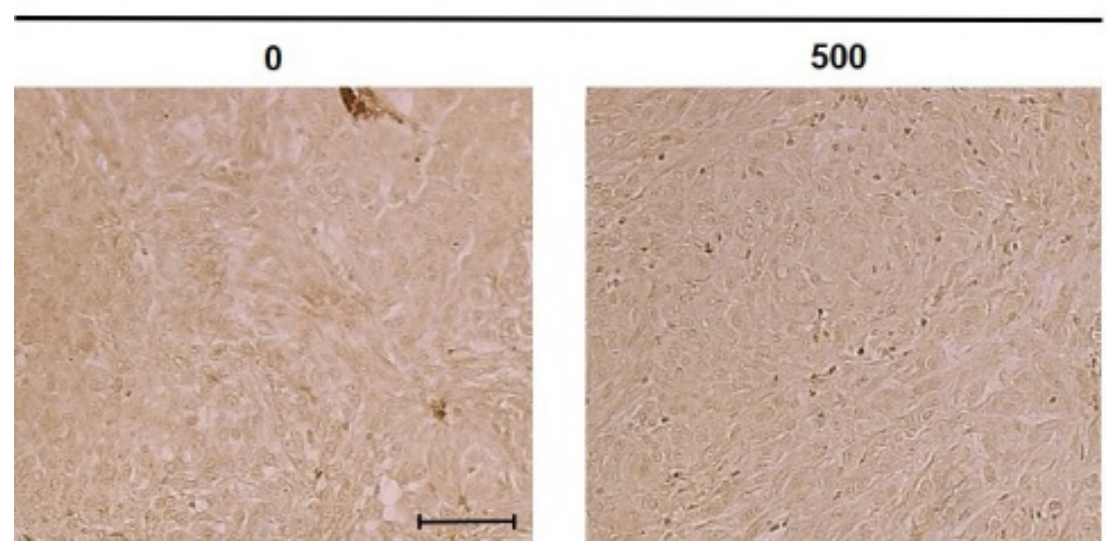

Figure 7. Effects of DPL on phospho (p)-p38 expression in lung cancer tumor tissues. Excised tumors were analyzed by immunohistochemistry with antibodies specific for p-p38. Slides were observed under a microscope $(200 \times)$. Scale bar, $10 \mu \mathrm{m}$

\section{DPL increases the expression of p-p38 in lung carcinoma tissues}

Immunohistochemistry was conducted to determine the effect of DPL on the expression of p-p38, an apoptosis-related protein, in xenografts of human lung carcinoma tissues. The number of p-p38positive cells was increased compared to the control group (Figure 7). Thus, administration of DPL induced apoptosis by increasing expression of p-p38 in A549 carcinoma cells.

\section{DPL does not cause histopathological changes in hepatic and kidney tissues}

To examine organ toxicity induced by administration of DPL, hepatic and kidney tissues of xenografted mice were stained with hematoxylin and eosin and observed using an optical microscope (Figure 8). No histopathological changes were apparent, suggesting DPL did not cause detectable toxicity.

\section{Discussion}

This study examined whether DPL induces apoptosis in A549 lung carcinoma cells as well as its anti-tumor effects in vivo. Prior to examining its effect on the viability of A549 cells, WB hepatic cells were treated with DPL to examine its effects in a normal cell line. Because viability was shown to decrease when cells were treated with $>600 \mu \mathrm{g} /$ $\mathrm{mL}$ DPL for $24 \mathrm{~h}$, cells were treated with $0-500 \mu \mathrm{g} / \mathrm{mL}$ in subsequent experiments. Further experiments revealed that the viability of A549 cells decreased following treatment with $\geq 300 \mu \mathrm{g} / \mathrm{mL}$ DPL in a concentration-dependent manner (Figure 1). According to Aceituno et al. [19], the viabilities of A549 lung carcinoma and HepG2 hepatic cells were reduced to 5 and $50 \mu \mathrm{g} / \mathrm{mL}$, respectively, when silver-nano particles of $D$. morbiferus were added at concentrations of $0,1,5,10$, and $50 \mu \mathrm{g} / \mathrm{mL}$ for $48 \mathrm{~h}$. According to Hyun et al. [9], the viability significantly decreased from $100 \mu \mathrm{g} / \mathrm{mL}$ when DPL was added to Huh7 liver carcinoma cells at concentrations of $0,12.5,25,50,100$, and $200 \mu \mathrm{g} /$ well for $72 \mathrm{~h}$. These findings are consistent with the results of this study, demonstrating the effect of DPL on the viability of various carcinoma cells.

As apoptosis occurs, DAPI and annexin $\mathrm{V}$ enter the nucleus through membrane permeabilization and stain the DNA, allowing visualization of apoptosis. Therefore, DAPI staining and flow cytometry were conducted to demonstrate the induction of apoptosis 


\section{DPL $(\mathrm{mg} / \mathrm{kg})$}

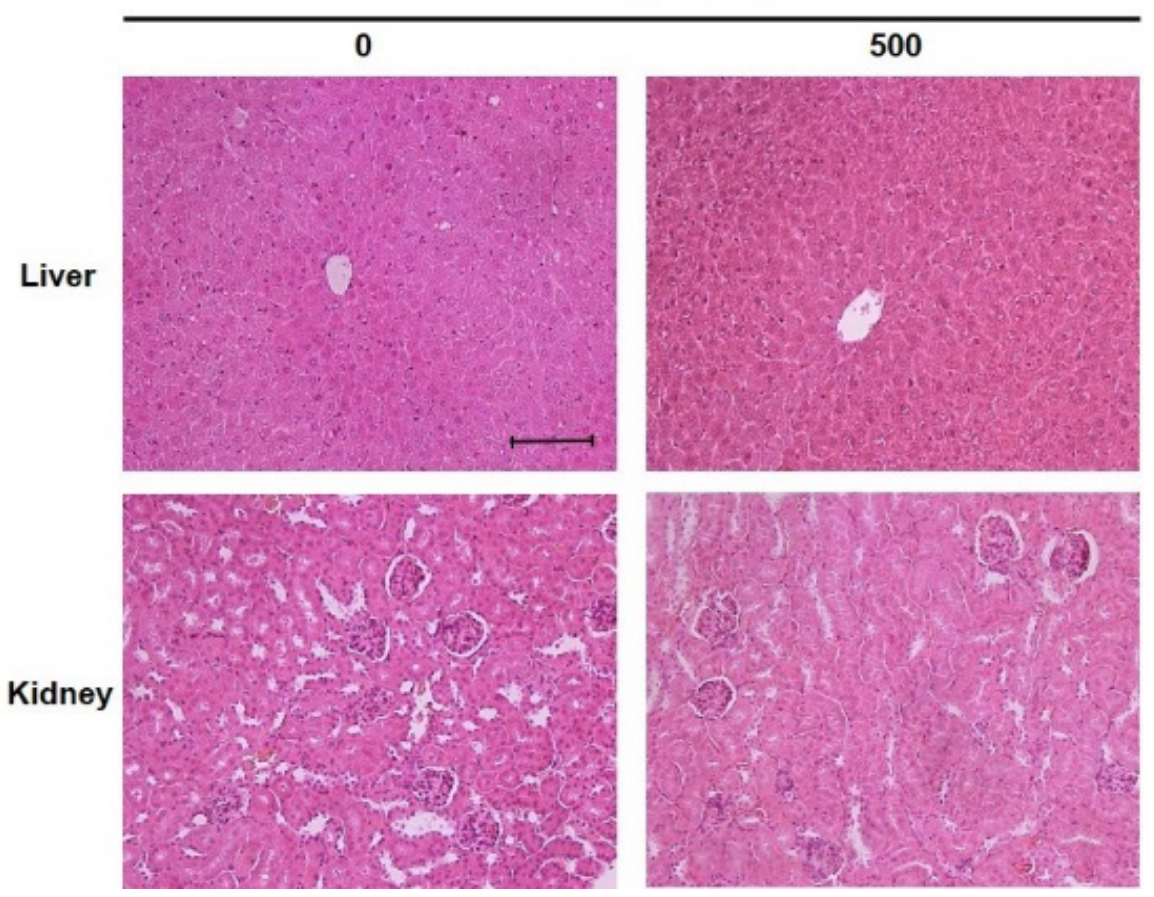

Figure 8. Mice received an injection of A549 cells. Livers and kidneys were then excised and evaluated by hematoxylin and eosin staining $(200 \times)$. Scale bar, $10 \mu \mathrm{m}$. DPL had no detectable toxic effects on the nude mice

following treatment of A549 carcinoma cells with DPL for $24 \mathrm{~h}$. DNA fragmentation and apoptotic bodies were observed through DAPI staining. Flow cytometry showed a concentration-dependent increase in apoptotic cells, consistent with the DAPI staining results. Lee et al. [20] observed concentration-dependent condensation of DAPI-stained chromatin following treatment of human myeloid leukemia U937 cells with a D. morbiferus stem extract at various concentrations for $24 \mathrm{~h}$. Hyun et al. [9] examined apoptosis using flow cytometry and reported an increase in the number of apoptotic human hepatoma Huh-7 cells following treatment with $50 \mu \mathrm{g}$ of yellow leaves and with $100 \mu \mathrm{g}$ of green leaves of D. morbiferus for $72 \mathrm{~h}$. These results indicate that the leaves and stem of D. morbiferus induce apoptosis and cause anti-tumor effects in various carcinoma cells.

In the present study, western blotting was performed to assess the levels of apoptosis-related proteins in DPL-treated A549 cells. Levels of the anti-apoptotic protein $\mathrm{Bcl}-2$ were decreased compared to the control group, while levels of the pro-apoptotic proteins Bax and cleaved PARP were increased. Im et al. [16] treated breast carcinoma MCF-7 and MDA-MB-231 cells with $0,100,150$, and $300 \mu \mathrm{g} / \mathrm{mL}$ of $D$. morbiferus stem extract for $24 \mathrm{~h}$ and examined apoptosis-related proteins. They observed that the expression of Bcl-2 and Bcl-xL was significantly decreased in MCF-7 cells following treatment with $300 \mu \mathrm{g} / \mathrm{mL}$ while the expression of Bad and Bax was significantly increased. In MDAMB-231 cells, the expression of Bcl-xL was significantly decreased following treatment with $300 \mu \mathrm{g} / \mathrm{mL}$, and the expression of Bax was significantly increased. Those authors observed that $D$. morbiferus leaf and stem extracts regulated $\mathrm{Bax}, \mathrm{Bcl}-2$, and PARP. This suggests that DPL induces apoptosis at the protein level.

When A549 cells were treated with DPL, expression levels of p-ERK1/2 and p-JNK were similar to the level in the control group and p-p38 increased in a concentration-dependent manner. Lee et al. [20] treated human myeloid leukemia U937 cells with $D$. morbiferus stem extract for specific time periods $(0,0.5,1,2,4,6,12$, and $24 \mathrm{~h})$ and examined MAPK pathway-related proteins by western blotting. They observed that p-ERK, $\mathrm{p}$-JNK, and p-p38 protein levels were increased at $0.5-6 \mathrm{~h}$. The present study observed a concentration-dependent increase in p-p38 when A549 cells were treated with DPL for $24 \mathrm{~h}$, whereas Lee et al. [20] observed an increase in p-p38 over 0.5-6 h followed by a decrease at $24 \mathrm{~h}$. These findings suggest that apoptosis was induced through the p38 MAPK pathway, because p-p38 increased in a concentration- and time-dependent manner in A549 cells when DPL was administered.

DPL was orally administered in mice to examine whether the in vivo anti-tumor effects were as significant as the effects in vitro. Tumor volume was inhibited beginning at day 15 after DPL administration and was significantly inhibited at day 22 . Tumor weight decreased by $22.2 \%$ in the DPL-administered group relative to the control group. Apoptosis was examined in A549 tumor tissue using the TUNEL assay; the results showed that the number of TUNEL-positive cells was increased compared with the control group. The number of p38-positive cells, a protein involved in the MAPK signaling pathway, was increased in tumor tissue compared with the control group.

In summary, this study showed the induction of apoptosis in A549 lung carcinoma cells by DPL both in vitro and in vivo through the p-p38 MAPK pathway. Through these anti-cancer effects, DPL may be a potential natural material for lung cancer treatment.

\section{Conclusion}

This study demonstrated the induction of apoptosis by the extract of D. morbifera leaves (DPL) through the MAPK pathway in A549 cells, 
a NSCLC cell line, and examined the effect of the extract on tumor growth in vivo. In conclusion, DPL decreased the viability of A549 cells and induced apoptosis by regulating p38 MAPK pathway in vitro. By examining in vivo tumor growth following administration of DPL, we showed that DPL significantly reduced tumor volume and increased apoptosis. DPL shows potential as a drug for lung cancer.

\section{Author' contributions}

Ji-Youn Jung and Eun-Seon Yoo conceptualized and designed the study. Eun-Seon Yoo, Sung-Hyun Kim, Joong-Seok Woo, Jae-Han Lee, So-Hee Han, and Soo-Hyun Jung acquired the data. Eun-Seon Yoo, JiYoun Jung, Sae Man Kim, Eun Gee Kim and Kwang Joong Kim analyzed and interpreted the data. Ji-Youn Jung and Eun-Seon Yoo drafted the manuscript. All authors have read and approved the final manuscript.

\section{Acknowledgment}

The present study was supported by Basic Science Research Program through the National Research Foundation of Korea (NRF) funded by the Ministry of Education, Science and Technology (grant no. NRF 2021R1A2C1010912).

\section{Conflict of interest}

The authors declare that they have no competing interests.

\section{References}

1. Statistics Korea (2017) Cause of death statistics. Last accessed on 23 September 2019.

2. National cancer information center. Last accessed on 23 September 2019.

3. Rafei H, El-Bahesh E, Finianos A, Nassereddine S, Tabbara I (2017) Immune-based therapies for non-small cell lung cancer. Anticancer Res 37: 377-387. [Crossref]

4. Park SB, Kim HN, Kim JD, Park GH, Eo HJ, et al. (2019) Inhibitory effect of the branch extracts from Taxillus yadoriki parasitic to Neolitsea sericea against the cell proliferation in human lung cancer cells, A549. Korean J Plant Res 32: 109-115.

5. Lin L, Cheng K, Xie Z, Chen C, Chen L, et al. (2019) Purification and characterization a polysaccharide from Hedyotis diffusa and its apoptosis inducing activity toward human lung cancer cell line A549. Int J Biol Macromol 122: 64-71. [Crossref]

6. Da Rocha AB, Lopes RM, Schwartsmann G (2001) Natural products in anticancer therapy. Curr Opin Pharmacol 1: 364-369.
7. Lee C, Yang M, Moon JO (2019) Antioxidant and hepatoprotective effects of the ethanol extract of Dendropanax morbifera Leveille on the t-butyl hydroperoxideinduced HepG2 cell damages. Korean J Pharmacogn 50: 32-36.

8. Lee SH, Lee HS, Park YS, Hwang B, Kim JH, et al. (2002) Screening of immune activation activities in the leaves of Dendropanax morbifera Lev. Korean J Med Crop Sci 10: 109-115.

9. Hyun TK, Kim MO, Lee H, Kim YJ, Kim EK, et al. (2013) Evaluation of anti-oxidant and anti-cancer properties of Dendropanax morbifera Léveille. Food Chem 141: 19471955.

10. Park SA, Park J, Park CI, Jie YJ, Hwang YC, et al. (2013) Cellular antioxidant activity and whitening effects of Dendropanax morbifera leaf extracts. J Microbiol Biotechnol 41: 407-415.

11. Lee MK, Lee IS, Lee JS (2013) For the utilization of native plant resources as highvalue materials: evaluation on demelanizing activity of Dendropanax morbifera in Bogildo. J Kor Island 25: 227-240.

12. Hyun TK, Ko YJ, Kim EH, Chung IM, Kim JS (2015) Anti-inflammatory activity and phenolic composition of Dendropanax morbifera leaf extracts. Ind Crops Prod 74: 263270

13. An NY, Kim JE, Hwang D, Ryu HK (2014) Anti-diabetic effects of aqueous and ethanol extract of Dendropanax morbifera Leveille in streptozotocin-induced diabetes model. J Nutr Health 47: 394-402.

14. Elmore S (2007) Apoptosis: a review of programmed cell death. Toxicol Pathol 35: 495-516.

15. Santarpia L, Lippman SM, El-Naggar AK (2012) Targeting the MAPK-RAS-RAF signaling pathway in cancer therapy. Expert Opin Ther Targets 16: 103-119.

16. Im KJ, Jang SB, Yoo DY (2015) Anti-cancer effects of Dendropanax Morbifera extract in MCF-7 and MDA-MB-231 cells. J Korean Obstet Gynecol 28: 26-39.

17. Kim SH, Choo GS, Yoo ES, Woo JS, Han SH, et al. (2019) Silymarin induces inhibition of growth and apoptosis through modulation of the MAPK signaling pathway in AGS human gastric cancer cells. Oncol Rep 42: 1904-1914.

18. Kim JM, Park JD, Park DC, Kim BO (2013) In vivo antitumor activity and acute, subacute toxicity of keumsa (Phellinus linteus) extracts. J Life Sci 23: 1388-1396.

19. Aceituno VC, Ahn S, Simu SY, Wang C, Mathiyalagan R, et al. (2016) Silver nanoparticles from Dendropanax morbifera Léveille inhibit cell migration, induce apoptosis, and increase generation of reactive oxygen species in A549 lung cancer cells. In Vitro Cell Dev Biol Anim 52: 1012-1019.

20. Lee JW, Park C, Han MH, Hong SH, Lee TK, et al. (2013) Induction of human leukemia U937 cell apoptosis by an ethanol extract of Dendropanax morbifera Lev. through the caspase-dependent pathway. Oncol Rep 30: 1231-1238. [Crossref]

Copyright: (C2021 Yoo ES. This is an open-access article distributed under the terms of the Creative Commons Attribution License, which permits unrestricted use, distribution, and reproduction in any medium, provided the original author and source are credited. 